\title{
ESI: Membrane-less photoelectrochemical cells: product separation by hydrodynamic control
}

Isaac Holmes-Gentle, Franca Hoffmann, Camilo A. Mesa and Klaus Hellgardt

April 3, 2017

\section{Contents}

1 Mathematical description of model and numerical solution 2

1.1 Constant electrode flux mass transfer model . . . . . . . . . . . . . . . . . 2

1.2 Multi-physics model . . . . . . . . . . . . . . . . . . 5

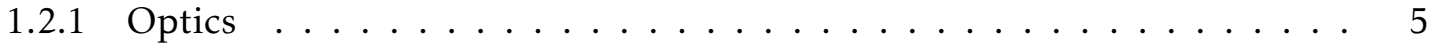

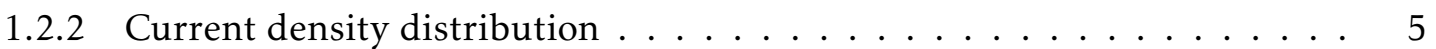

1.2 .3 Transport equation $\ldots \ldots \ldots \ldots \ldots \ldots \ldots$

1.3 Discretisation details $\ldots \ldots \ldots \ldots \ldots \ldots \ldots$

2 Separator position in cell $\quad 8$

3 Experimental details $\quad 8$

3.1 Calculation of effective interfaces $\ldots \ldots \ldots \ldots$

3.1.1 Optics of photo-anode and cathode . . . . . . . . . . . . . 9

4 Electrode current density distribution $\quad 11$

5 Predicted photocurrent behaviour from IPCE integration $\quad 12$

6 References 12 


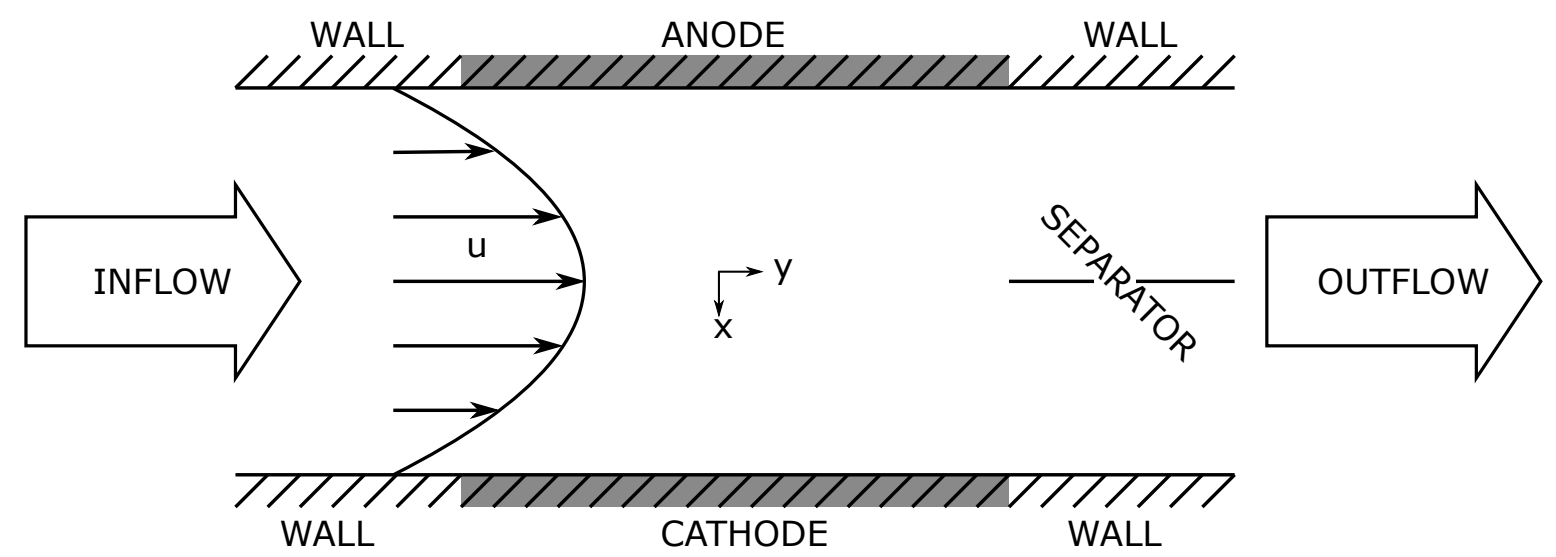

Figure 1: Geometry of cell design

\section{Mathematical description of model and numerical solution}

\subsection{Constant electrode flux mass transfer model}

$$
D_{i} \frac{\partial^{2} c_{i}}{\partial x^{2}}+D_{i} \frac{\partial^{2} c_{i}}{\partial y^{2}}-u_{y} \frac{\partial c_{i}}{\partial y}=0
$$

The diffusion-convection equation for species i at steady state is given by eq. (1), where the the only non-zero component of the fluid velocity $\boldsymbol{u}=\left(u_{x}, u_{y}\right)$ is the y component. The diffusivity of species i $D_{i}$ is a positive constant. The flow profile $u_{y}$ is an analytic function given by equation (2).

$$
u_{y}=\frac{1}{2 \mu} \Delta P_{d r o p}\left(L_{x} x-x^{2}\right)
$$

The boundary conditions for species i are given in eq. (3) - (7). They can also be seen in Figure $2 \mathrm{a}$ where for each species on one electrode they are produced and the other they are consumed at a rate fast enough to assume $c_{i}=0$.

$$
\begin{array}{rlrl}
c_{i} & =c_{i, 0} & \text { on inlet } \\
\frac{\partial c_{i}}{\partial y} & =0 & & \text { on outlet } \\
-\left.\vec{n} \cdot N_{i}\right|_{x=0 \text { or } L_{x}} & =0 & & \text { on walls } \\
-\left.\vec{n} \cdot N_{i}\right|_{x=0 \text { or } L_{x}} & =R_{i, e l e c t r o d e} & & \text { on producing electrode } \\
c_{i} & =0 & & \text { on consuming electrode }
\end{array}
$$

As $u_{x}=0$, the boundary conditions that are specified as a flux, equations (6) - (7), can be rearranged to neumann boundary conditions for concentration to the surface. 


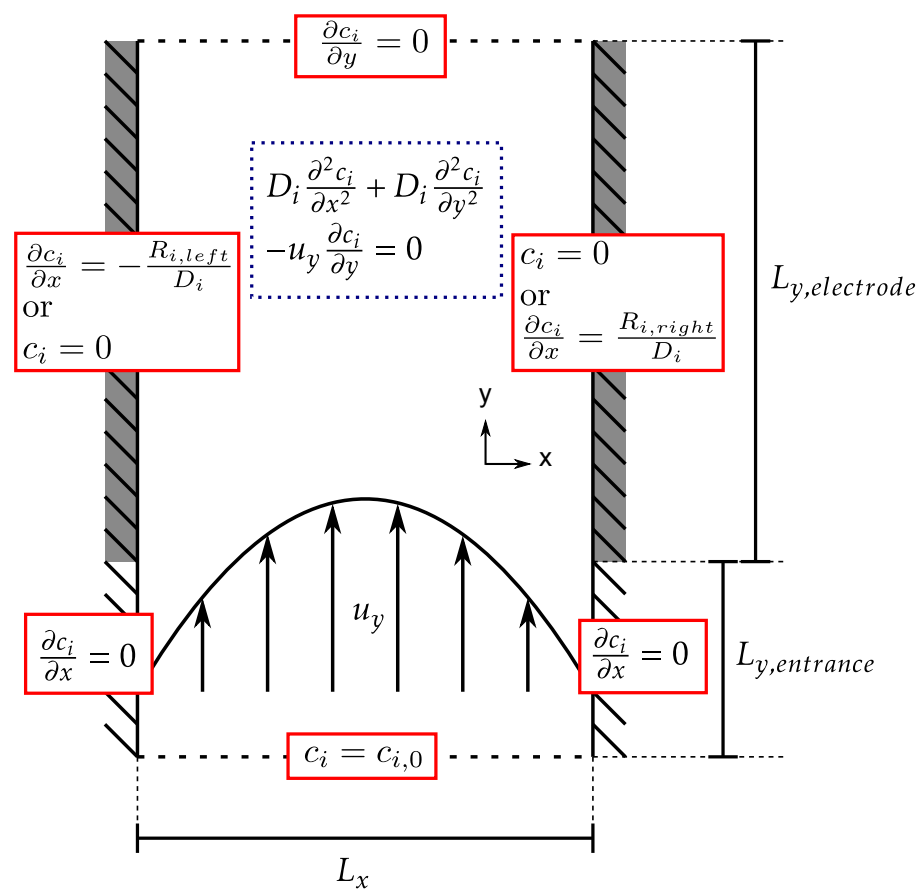

(a) Mass transfer

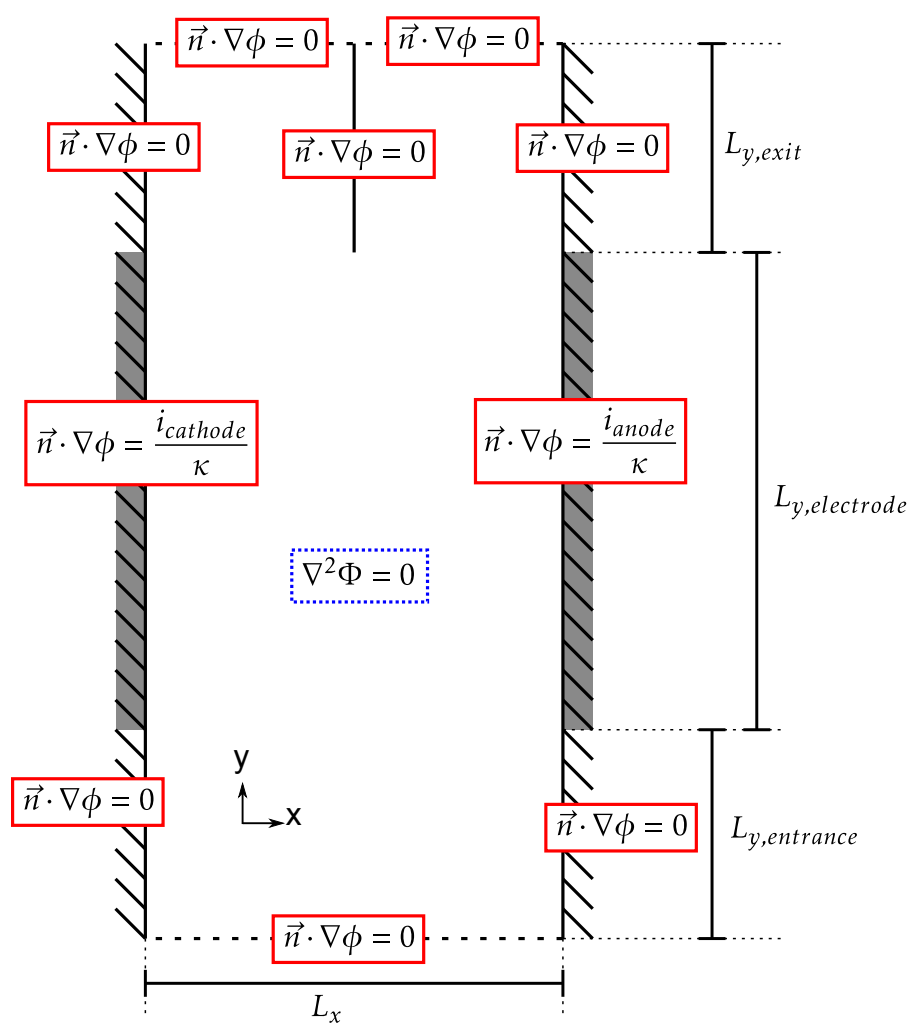

(b) Current density distribution

Figure 2: Diagrams showing boundary conditions 


$$
\begin{array}{ll}
\text { Left: } & \left.\frac{\partial c_{i}}{\partial x}\right|_{x=0}=\left\{\begin{array}{lr}
0 & \text { on insulating wall } \\
-R_{i, \text { left }} / D_{i} & \text { on electrode }
\end{array}\right. \\
\text { Right: } & \left.\frac{\partial c_{i}}{\partial x}\right|_{x=L_{x}}=\left\{\begin{array}{lr}
0 & \text { on insulating wall } \\
R_{i, \text { right }} / D_{i} & \text { on electrode }
\end{array}\right.
\end{array}
$$

Since the concentration $c_{i}$ for each species $\mathrm{i}$ is modelled by an equation of the same form, we denote for the rest of this section $c=c_{i}, u=u_{y}$ in order to simplify notation. We use a space discretisation $x_{0}, \cdots, x_{n_{x}}$ and $y_{0}, \cdots, y_{n_{y}}$ where $x_{i}=i \Delta x$ for each $i \in\left\{0, \cdots, n_{x}\right\}$ and $y_{j}=j \Delta y$ for each $y \in\left\{0, \cdots, n_{y}\right\}$. Then the dimensions of the channel are given by $L_{x}=n_{x} \Delta x$ and $L_{y}=n_{y} \Delta y$. With this notation, the model (1) for the concentration $c_{i, j}=c\left(x_{i}, y_{j}\right)$ can be discretised as eq. (10) - (12). The 2 nd order derivative with respect to $\mathrm{x}$ and $\mathrm{y}$ are discretised with $2 \mathrm{nd}$ order body centred finite difference schemes (eq. (10) \& (11)). For $\frac{\partial c}{\partial y}$, a 2nd order upwinding scheme is used since the velocity in the y direction $\left(u_{y}\right)$ is always strictly positive.

$$
\begin{aligned}
\frac{\partial^{2} c}{\partial x^{2}} & =\frac{c_{i+1, j}-2 c_{i, j}+c_{i-1, j}}{(\Delta x)^{2}} \\
\frac{\partial^{2} c}{\partial y^{2}} & =\frac{c_{i+1, j}-2 c_{i, j}+c_{i-1, j}}{(\Delta y)^{2}} \\
\frac{\partial c}{\partial y} & =\frac{3 c_{i, j}-4 c_{i, j-1}+c_{i, j-2}}{2 \Delta y}
\end{aligned}
$$

The boundary conditions are discretised as follows:

$$
\begin{array}{r}
\left.c\right|_{x=0}=c_{0, j} \\
\left.c\right|_{x=L_{x}}=c_{n_{x}, j} \\
\left.\frac{\partial c}{\partial x}\right|_{x=0}=\frac{c_{2, j}-c_{0, j}}{2 \Delta x} \\
\left.\frac{\partial c}{\partial x}\right|_{x=L_{x}}=\frac{c_{n_{x}+1, j}-c_{n_{x}+1, j}}{2 \Delta x} \\
\left.\frac{\partial c}{\partial y}\right|_{y=L_{y}}=\frac{c_{y=0}=c_{i, 0}}{2 \Delta x}
\end{array}
$$

The space was discretised onto a grid and the resulting sparse matrices are solved using a multi-purpose inbuilt linear solver (MATLAB).

It is important to note that for the mass transfer model, the exit of the channel is not simulated (seen in Fig. 2a). This is because 1) the concentration distribution at the point the fluid is separated into two is the parameter of interest 2) the velocity of fluid can in this region can 
be approximated by an analytic function of $x$ (see eq. 2). The second important point to note is that the outflow condition is only valid if the solution down stream does not affect the solution upstream of the boundary. However, the diffusion counterflow will be far smaller than the transport via convection out of the cell (by virtue of the fact the products are being hydrodynamically separated). Therefore, this error is not significant in terms of the outcomes found.

\subsection{Multi-physics model}

\subsubsection{Optics}

The transfer matrix method is implemented by first defining the structure of the optical system and for each layer $(k \in\{1, \cdots, m\})$ a complex refractive index $\left(\tilde{n}_{k}\right)$ and layer thickness $\left(d_{k}\right)$ given. From this, the $2 \times 2$ layer and interface matrices are calculated. Then the system matrix $\bar{S}$ can be determined and overall transmittance, reflectance and absorptance computed. Layer absorptance is calculated by stepping through each layer in the stack and calculating the power absorbed. This methodology is repeated for each wavelength over the wavelength range of interest. Byrnes [1] gives an extensive description of how to implement the transfer matrix model.

One important consideration on the stability of the transfer matrix method is that, as the layer widths increases, the amount of light passing through that layer is reduced exponentially. This means the layer matrix tends to equation (20).

$$
L_{k}=\left[\begin{array}{cc}
\infty & 0 \\
0 & 0
\end{array}\right] \quad \text { as } d_{k} \rightarrow \infty
$$

This can cause computational issues so $L^{1,1}$ where $L^{i, j}$ denotes the $\left(i^{\text {th }}, j^{\text {th }}\right)$ entry of the $k^{\text {th }}$ layer matrix $L_{k}$ in the stack is replaced by some arbitrary large number which is large enough to keep numerical errors small.

Secondly, when the stack is completely absorbing, the system transfer matrix becomes illconditioned. Therefore it becomes difficult to accurately solve the problem for when light is shined simultaneously in to both ends of the stack. A work-around is to only solve for light in the forward direction, and to flip the stack in order to solve the reverse problem 'forward'. As the light from either end of the stack is assumed to be incoherent, the total optical power flow in the stack can be calculated through summation.

\subsubsection{Current density distribution}

A secondary current density distribution model was employed which includes the electrode kinetics but not the mass-transfer effects [2]. As the current density is significantly lower than the limiting current that the electrolyte can support this is a valid methodology.

$$
\nabla^{2} \Phi=0 \quad i=-\kappa \nabla \Phi
$$


The boundary conditions come from specifying the current density across the boundary. Either the boundary is insulating $(-\overrightarrow{\boldsymbol{n}} \cdot \boldsymbol{i}=0)$ or it allows the flow of charge across it $\left(-\overrightarrow{\boldsymbol{n}} \cdot \boldsymbol{i}=i_{\text {electrode }}\right)$ where the electrode current density $i_{\text {electrode }}$ is a function of the electrode potential $E$ which is defined as $E=\Phi_{\text {electrode }}-\Phi(y)+$ constant) on the electrode-electrolyte interface. Here the constant is arbitrary and comes from the type reference electrode system used, $\Phi_{\text {electrode }}$ is the absolute electric potential of the electrode and is constant and $\Phi(y)$ is the absolute electric potential of the electrolyte at the interface and is dependant on the position on the interface $(y)$.

Given $\boldsymbol{i}=-\kappa \nabla \Phi$, this becomes $\overrightarrow{\boldsymbol{n}} \cdot \nabla \phi=0$ for the insulating boundaries (walls, inlet, outlet, separator). At the electrodes, $\overrightarrow{\boldsymbol{n}} \cdot \nabla \phi=i_{\text {electrode }} / \kappa$. A diagram of the boundary conditions for the current density distribution can be seen in Figure $2 b$.

Using the same notation as was used in the discretisation of the constant flux mass transfer model, the laplace equation is discretised using a 2nd order centred finite difference scheme as shown in eq. (22). The boundary conditions for this are given by eq. (23) - (26).

$$
\begin{aligned}
0 & =\frac{\Phi_{i+1, j}-2 \Phi_{i, j}+\Phi_{i-1, j}}{(\Delta x)^{2}}+\frac{\Phi_{i, j+1}-2 \Phi_{i, j}+\Phi_{i, j-1}}{(\Delta y)^{2}} \\
\left.\frac{\partial \Phi}{\partial x}\right|_{x=0} & =\frac{\Phi_{2, j}-\Phi_{0, j}}{2 \Delta x} \\
\left.\frac{\partial \Phi}{\partial x}\right|_{x=L_{x}} & =\frac{\Phi_{n_{x}+1, j}-\Phi_{n_{x}-1, j}}{2 \Delta x} \\
\left.\frac{\partial \Phi}{\partial y}\right|_{x=0} & =\frac{\Phi_{i, 2}-\Phi_{i, 0}}{2 \Delta y} \\
\left.\frac{\partial \Phi}{\partial y}\right|_{x=L_{y}} & =\frac{\Phi_{i, n_{y}+1}-\Phi_{i, n_{y}-1}}{2 \Delta y}
\end{aligned}
$$

The kinetics of the electrode gives the voltage current relationship.

- Cathode: Bulter-Volmer

$$
i_{\text {cathode }}(E)=i_{\circ}\left(e^{\alpha_{a} f\left(E-E^{e q}\right)}-e^{-\alpha_{c} f\left(E-E^{e q}\right)}\right)
$$

Where the electrode potential $E$ of the interface is a function of position on the surface of the electrode, hence $E(y) . \alpha_{a}, \alpha_{c}, f, E^{e q}$ and $i_{0}$ are all constants as defined in the list of symbols in the main text.

- Photo-anode: Integrated IPCE vs E relationship

$$
i_{\text {anode }}(E)=\int_{0}^{\infty} e\left(\operatorname{IPCE}^{\mathrm{f}}(E, \lambda) q_{p}^{\mathrm{f}}(\lambda)+\operatorname{IPCE}^{\mathrm{b}}(E, \lambda) q_{p}^{\mathrm{b}}(\lambda)\right) d \lambda
$$

Where $I P C E^{i}, q_{p}^{i}$ is the incident photon to current efficiency and the spectral photon flux respectively where the superscript $i$ gives the direction of illumination ( $\mathrm{f}$ for forward and $\mathrm{b}$ for backward). The spectral photon flux $q_{p}$ vanishes for small wavelengths of light and 
Table 1: Numerical solution details (Where N.D.A is Non-Dimensional Analysis)

\begin{tabular}{|c|c|c|c|}
\hline & Model & Symbol & Value/Dataset/Notes \\
\hline \multirow{4}{*}{ 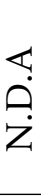 } & \multirow{4}{*}{ Mass transfer } & $n_{x}$ & 401 \\
\hline & & $n_{y}$ & 401 \\
\hline & & $L_{y, \text { entrance }}$ & $=0.5 \times L_{x}$ \\
\hline & & $L_{y}^{t o t}$ & $=L_{y, \text { entrance }}+L_{y, \text { electrode }}$ \\
\hline \multirow{13}{*}{ 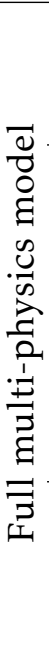 } & \multirow{3}{*}{ Optics } & $\lambda_{\text {start }}$ & $310 \mathrm{~nm}$ \\
\hline & & $\lambda_{\text {end }}$ & $1000 \mathrm{~nm}$ \\
\hline & & $\Delta \lambda$ & $1 \mathrm{~nm}$ \\
\hline & \multirow{7}{*}{ Current density model } & $\lambda_{\text {start }}$ & $310 \mathrm{~nm}$ \\
\hline & & $\lambda_{\text {end }}$ & $600 \mathrm{~nm}$ \\
\hline & & $\Delta \lambda$ & $1 \mathrm{~nm}$ \\
\hline & & $L_{y, \text { entrance }} / L_{v}^{\text {tot }}$ & 0.2 \\
\hline & & $L_{y, \text { exit }} / L_{y}^{\text {tot }}$ & 0.3 \\
\hline & & $n_{x}$ & 148 \\
\hline & & $n_{y}$ & 148 \\
\hline & \multirow{3}{*}{ Mass transfer } & $n_{x}$ & 101 \\
\hline & & $n_{y}$ & 101 \\
\hline & & $\begin{array}{l}L_{y, \text { entrance }} \\
L_{v}^{\text {tot }}\end{array}$ & $\begin{array}{l}=0.5 \times L_{x} \\
=L_{v, \text { entrance }}+L_{v, \text { electrode }}\end{array}$ \\
\hline
\end{tabular}

IPCE vanishes for large wavelengths of light (and also for $q_{p}$ but for a $\lambda$ greater) meaning that this integral is accurately approximated by some interval $\left[\lambda_{\text {start }}, \lambda_{\text {end }}\right]$.

The relationship between IPCE and $E$ is measured experimentally. Then for a given electrode potential $E$, the value for IPCE can be interpolated from the experimental data.

The boundary conditions at the electrodes leads to a non-linear system, which can be solved using a Newton-Raphson procedure (MATLAB).

\subsubsection{Transport equation}

The transport equation is discretised and solved in much the same fashion as in the masstransfer only model. The only difference is that now $R_{i \text {,left/right }}$ is a function of $y$ which is calculated from the solution of the secondary current density model $\left(i_{\text {electrode }}\right)$ by eq. (29).

$$
R_{i, \text { left } / \text { right }}=\frac{\eta_{\text {faradiac }} i_{\text {left } / \text { right }}}{n_{e^{-}} F}
$$

\subsection{Discretisation details}

Details of the discretisation of the dimensions $x, y$ and $\lambda$ are given in Table 1 . This also includes the length of any entrance or exit. For the optic component of the multi-physics model, the interval $\left[\lambda_{\text {start }}, \lambda_{\text {end }}\right]$ and interval spacing $\Delta \lambda$ refer to the set of wavelengths at which the transfer matrix model was solved for. For the current density model, these was parameters refer to the discretisation of the integration given in eq. (28). 


\section{Separator position in cell}

For the basic investigation of the given cell design the separator was placed in the middle of the cell (at $L_{x} / 2$ ). However this is not necessarily the optimal position for it as $D_{\mathrm{H}_{2}}$ and $D_{\mathrm{O} 2}$ equals $4.5 \times 10^{-9}$ and $2.1 \times 10^{-9} \mathrm{~m}^{2} \mathrm{~s}^{-1}$ respectively and the water splitting stoichiometry means the molar flux of $\mathrm{H}_{2}$ is twice that of $\mathrm{O}_{2}$.

The effect of this can be seen in Figure 3 for a given set of model parameters where the separator position at which highest collection efficiency is achieved is at $x / L_{x}=0.420$. It is positioned to the left of the centre as hydrogen has both a higher diffusivity and molar production rate.

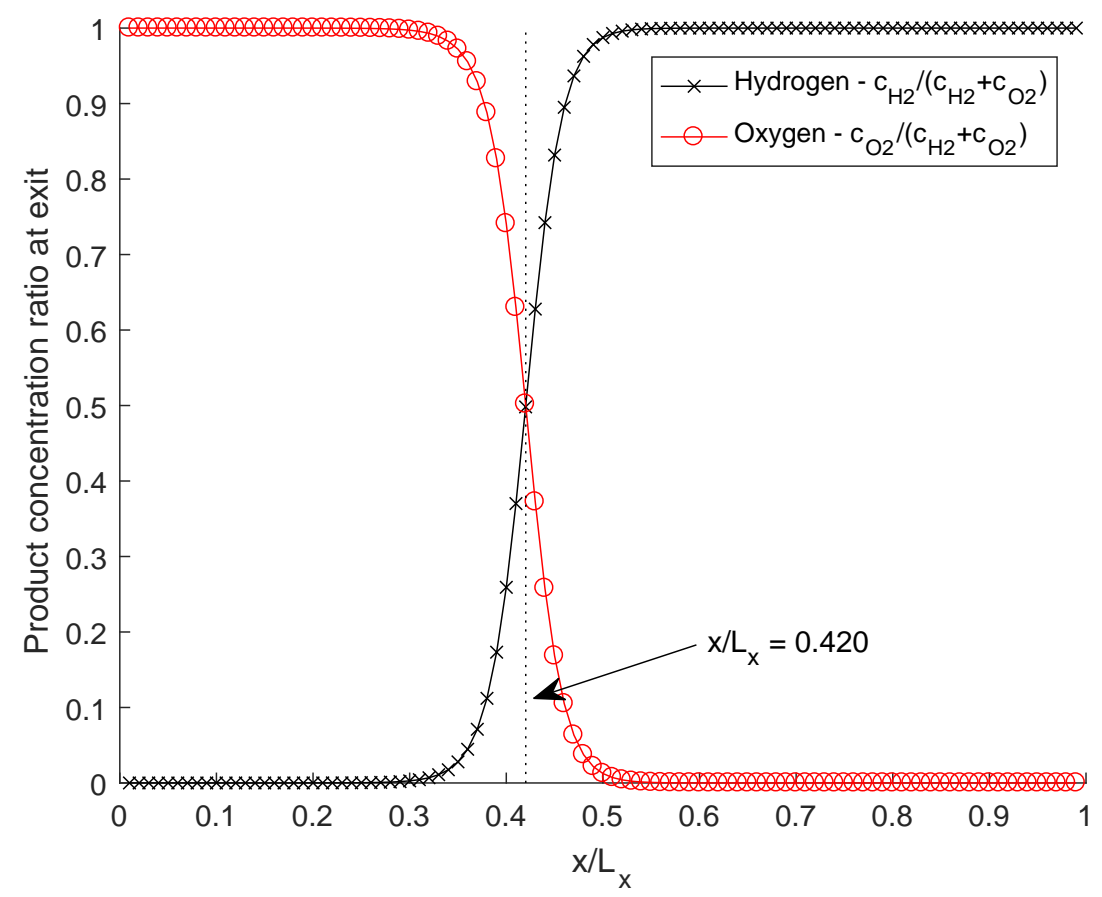

Figure 3: Product concentration ratio at the outlet of the cell from the mass transfer only model. The key model parameters are $L_{x}=5 \mathrm{~mm}, L_{y}$,electrode $=10 \mathrm{~mm},\left\langle u_{y}\right\rangle=10 \times 10^{-5} \mathrm{~ms}^{-1}$, $D_{H_{2}}=4.5 \times 10^{-9} \mathrm{~m}^{2} \mathrm{~s}^{-1}$ and $D_{\mathrm{O} 2}=2.1 \times 10^{-9} \mathrm{~m}^{2} \mathrm{~s}^{-1}$

\section{Experimental details}

\subsection{Calculation of effective interfaces}

In order to measure the optics of the glass : thin film : electrolyte interface a test cell was made by stacking a $1 \mathrm{~mm}$ silicone gasket and a microscope coverslip (soda lime glass) on top. The optics of this cell was represented in the transfer matrix model by the diagram in Figure 4.

The forward and backwards transmittance and total reflectance was determined by a UV-vis spectrophotometer with an integrating sphere attachment.

From this, the system intensity transfer matrix $\bar{S}$ could be calculated (eq. (30)) using the intensity reflection and transmittance coefficients for forward and backward illumination for 


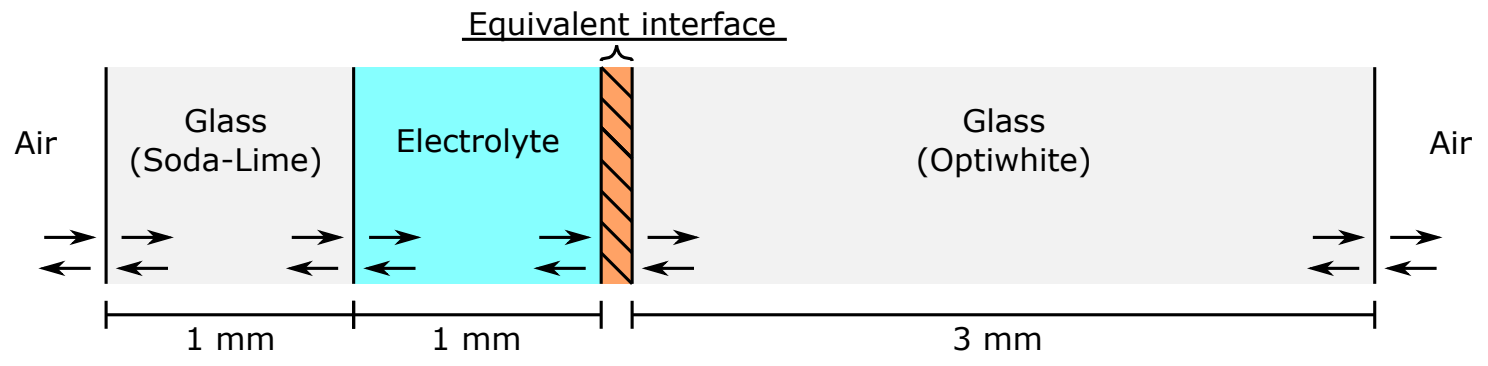

Figure 4: Optical geometry of the test cell

the whole stack. The intensity reflection $\bar{r}^{+/-}$and transmission $\bar{t}^{+/-}$coefficients can be related to the total reflectance $R^{+/-}$and transmittance $T^{+/-}$by equation (31) where the superscript denotes the forward $(+)$ and backward $(-)$ propagating electromagnetic waves.

$$
\begin{gathered}
\overline{\boldsymbol{S}}=\frac{1}{\bar{t}^{+}}\left[\begin{array}{cc}
1 & -\bar{r}^{-} \\
\bar{r}^{+} & \bar{t}^{+} \bar{t}^{-}-\bar{r}^{+} \bar{r}^{-}
\end{array}\right] \\
R^{+}=\bar{r}^{+} \quad T^{+}=\bar{t}^{+} \frac{\operatorname{Real}\left\{\tilde{n}_{j+1}\right\}}{\tilde{n}_{j}} \quad R^{-}=\bar{r}^{-} \quad T^{-}=\bar{t}^{-} \frac{\operatorname{Real}\left\{\tilde{n}_{j}\right\}}{\tilde{n}_{j+1}}
\end{gathered}
$$

The intensity system matrix is matrix multiplication of all the intensity interface matrices and intensity layer matrices in order of the stack (eq. (32)). Therefore if all the complex refractive indices are known for all the layers except the equivalent interface (see Figure 4), then the equivalent interface matrix is given by equation (33).

$$
\begin{gathered}
\overline{\boldsymbol{S}}=\overline{\boldsymbol{I}}_{0,1} \overline{\boldsymbol{L}}_{1} \overline{\boldsymbol{I}}_{1,2} \cdots \overline{\boldsymbol{L}}_{j} \overline{\boldsymbol{I}}_{j, j+1}^{\text {equiv }} \overline{\boldsymbol{L}}_{j+1} \cdots \overline{\boldsymbol{L}}_{m} \overline{\boldsymbol{I}}_{m, m+1} \\
\overline{\boldsymbol{I}}_{j, j+1}^{\text {equiv }}=\left(\overline{\boldsymbol{I}}_{0,1} \overline{\boldsymbol{L}}_{1} \overline{\boldsymbol{I}}_{1,2} \cdots \overline{\boldsymbol{L}}_{j}\right)^{-1} \overline{\boldsymbol{S}}\left(\overline{\boldsymbol{L}}_{j+1} \cdots \overline{\boldsymbol{L}}_{m} \overline{\boldsymbol{I}}_{m, m+1}\right)^{-1}
\end{gathered}
$$

The intensity reflection and transmission coefficients for the equivalent interface now can be calculated from:

$$
\bar{r}^{+}=\frac{\bar{S}_{21}}{\bar{S}_{11}} \quad \bar{t}^{+}=\frac{1}{\bar{S}_{11}} \quad \bar{r}^{-}=-\frac{\bar{S}_{12}}{\bar{S}_{11}} \quad \bar{t}^{-}=\frac{\operatorname{det}(\overline{\mathcal{S}})}{\bar{S}_{11}}
$$

The assumption that the glass coverslip was made of soda lime glass was found to be accurate enough for the purpose of this model. This was done by taking literature values for the complex refractive index for soda lime glass [3] and comparing the model outputs to the transmittance and reflectance of the glass coverslip only. The result of this can be seen in Figure 5.

\subsubsection{Optics of photo-anode and cathode}

For the calculation of the equivalent interface matrix for the photo-anode, there was slightly higher predicted reflectance from the glass optiwhite than was measured for the stack at 


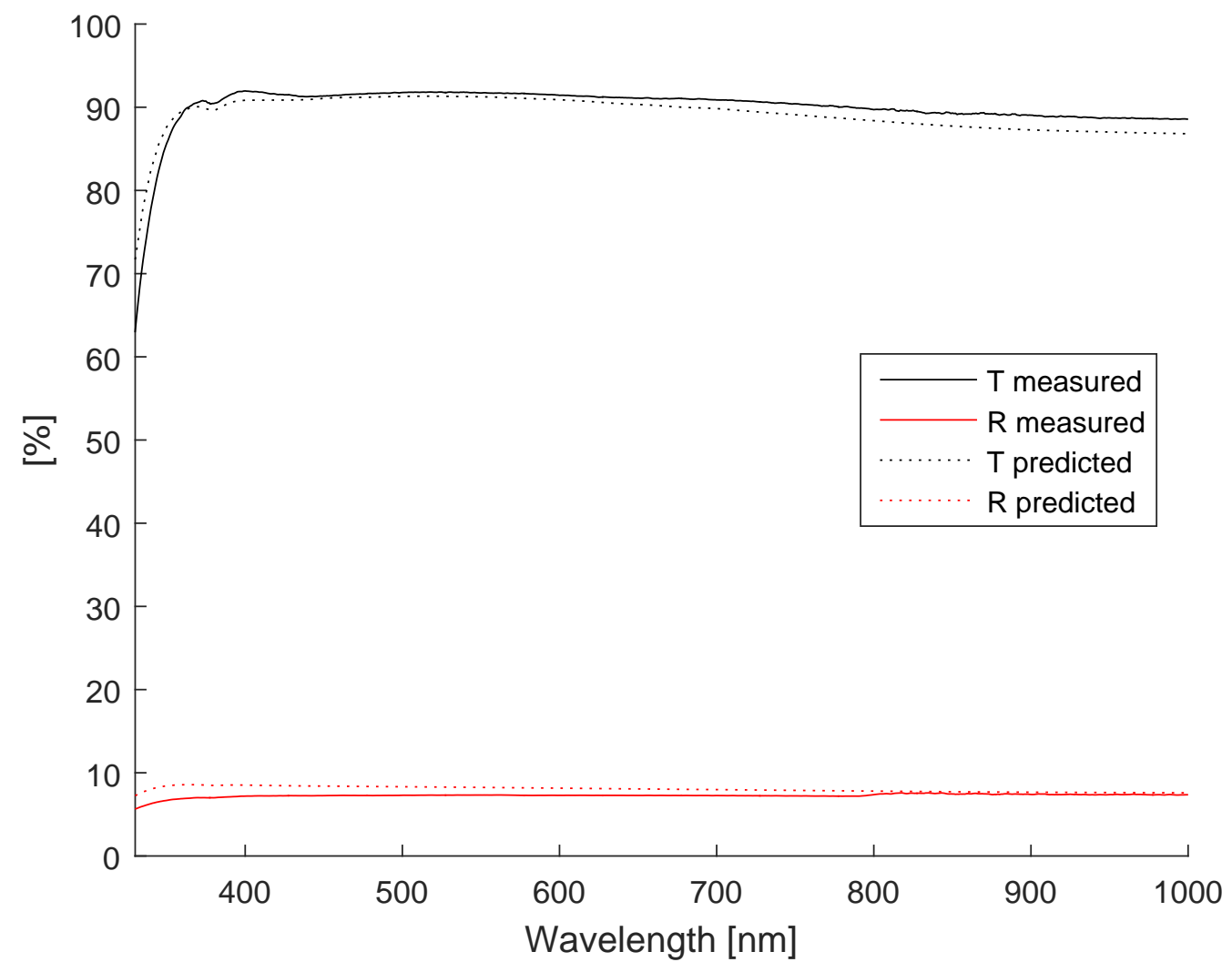

Figure 5: Measured and calculated transmittance and reflectance of the glass coverslip only showing the accuracy of the soda lime glass nk data 
wavelengths lower than $335 \mathrm{~nm}$, leading to a equivalent interface $\mathrm{R}^{-}$value that was negative. This failure of the model (specifically optiwhite nk data) to match reality is negligible as: 1) deviation is small (difference in reflectance between the experimental data for the test cell and predicted results for optiwhite glass alone is smaller than $0.3 \%$ for the $310-335 \mathrm{~nm}$ region) 2) This error is for the negative direction (glass-photoanode-electrolyte) and the solar illumination is in the opposite direction and so the small fraction that is reflected throughout the stack is subject to this error.

\section{Electrode current density distribution}

As seen in Figure 6, the current density distribution on the surface of the electrode is negligible and hence the assumption of constant electrode reaction flux is accurate.

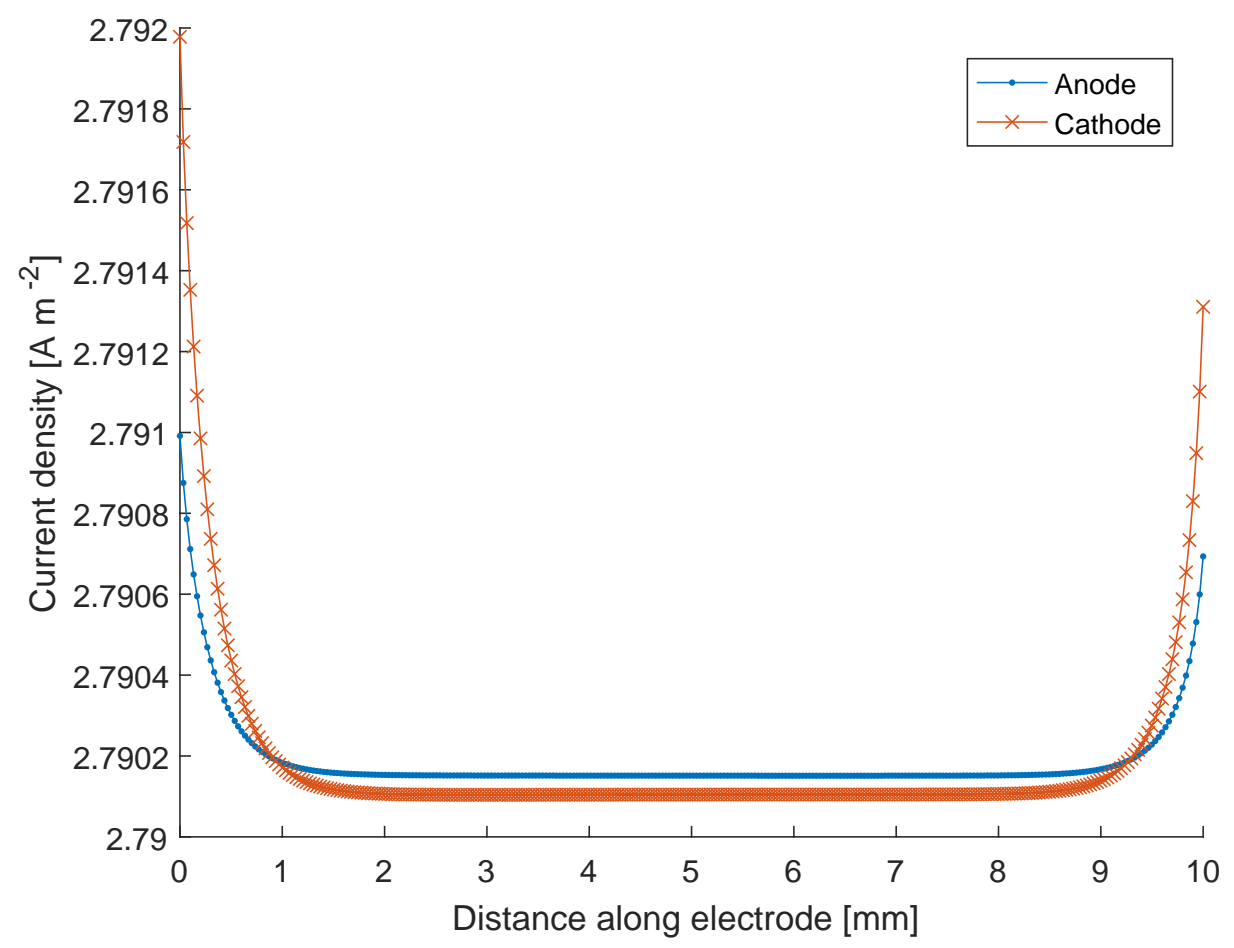

Figure 6: Electrode current density from the multiphysics model with $L_{x}=1 \mathrm{~mm}, L_{y, \text { electrode }}=10$ $\mathrm{mm}$ and $V_{\text {bias }}=1.4 \mathrm{~V}$ 


\section{Predicted photocurrent behaviour from IPCE integration}

Figure 7 shows an example of the predicted photocurrent vs electrode potential behaviour of the photo-anode using the experimentally determined IPCE data and the calculated irradiance at the surface of the electrode assuming an optical geometry (air-optiwhite-water- $\mathrm{Fe}_{2} \mathrm{O}_{3}$-optiwhiteair).

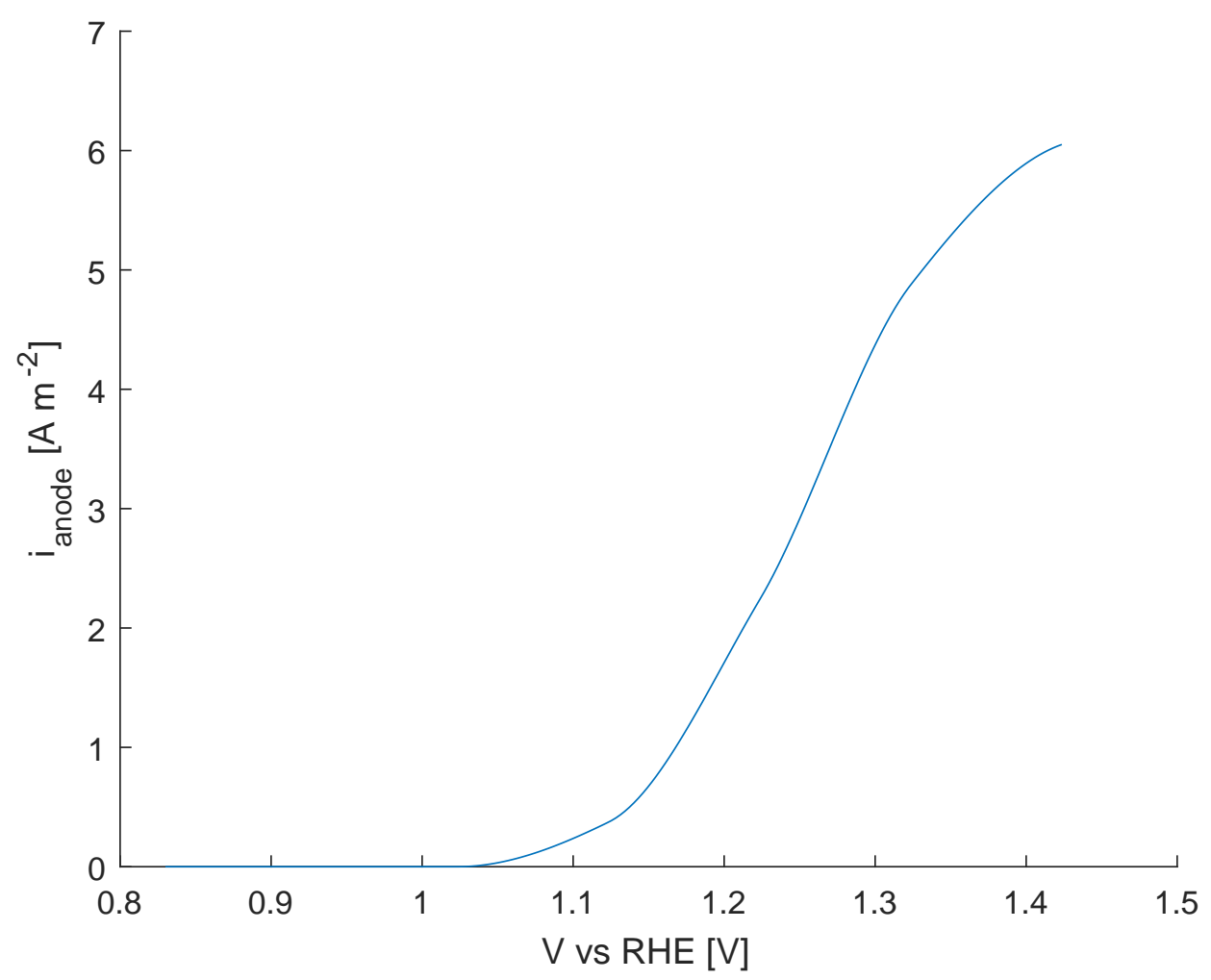

Figure 7: Integrated IPCE curve at various electrode potentials for AM1.5 illuminating airoptiwhite-water- $\mathrm{Fe}_{2} \mathrm{O}_{3}$-optiwhite-air for a channel width $L_{x}=1 \mathrm{~mm}$

\section{References}

[1] S. J. Byrnes. "Multilayer Optical Calculations". In: ArXiv e-prints (Mar. 2016), arXiv:1603.02720v2.

[2] John Newman and Karen E Thomas-Alyea. Electrochemical Systems. John Wiley \& Sons, 2012.

[3] M. Rubin. "Optical Properties of Soda Lime Silica Glasses". In: Solar Energy Materials 12.4 (1985), pp. 275-288. 